\title{
Methylmalonic Acid and Homocysteine in Plasma as Indicators of Functional Cobalamin Deficiency in Infants on Macrobiotic Diets
}

\author{
J. SCHNEEDE, P. C. DAGNELIE, W. A. van STAVEREN, S. E. VOLLSET, H. REFSUM,
} AND P. M. UELAND

Department of Pharmacology and Toxicology, University of Bergen, Haukeland Hospital, N-5021 Haukeland, Norway [J.S., H.R., P.M.U.|; Institute of Internal Medicine II, Erasmus University of Rotterdam, 3000 DR Rotterdam, The Netherlands /P.C.D./; Department of Human Nutrition, Agricultural University of Wageningen, $6700 \mathrm{EV}$ Wageningen, The Netherlands [W.A.v.S.]; and Section for Medical Informatics and Statistics, University of Bergen, N-5021 Haukeland, Nonway [S.E.V.]

\section{ABSTRACT}

\begin{abstract}
Methylmalonic acid and total homocysteine in plasma and serum have previously been used as indicators of intracellular cobalamin function in adults. To assess the usefulness of quantitation of these metabolites in the diagnosis of dietary cobalamin deficiency in infants, they were determined in plasma from 41 infants (aged $10-20 \mathrm{mo}$ ) on a macrobiotic dict and in 50 healthy group-matched omnivorous controls. In the macrobiotic infants, both methylmalonic acid and total homocysteine were markedly increased compared with controls (8-fold and 2-fold, respectively). Both metabolites showed an inverse relation to the plasma cobalamin level. The very low cobalamin content of the macrobiotic diet and low plasma cobalamin in macrobiotic infants makes an impaired cobalamin function likely in these infants. We therefore used dietary group as an independent indicator of cobalamin status. Different test parameters for cobalamin status were evaluated by comparing their ability to discriminate between the two dietary groups. Logistic regression analysis showed that methylmalonic acid followed by total homo-
\end{abstract}

The human requirement for cobalamin is usually covered by food of animal origin (1). Nutritional cobalamin deficiency may, however, develop in strict vegetarians $(1,2)$. Of particular concern are several reports on severe cobalamin deficiency in infants born to vegetarian mothers (2-6). Neurologic disorders have been observed in such infants (7).

Megaloblastic anemia and low serum cobalamin have long been considered the mainstay in the diagnosis of

Received Octoher 25, 1993; accepted lebruary 24, 1904

(orrespondence: Jorn Schnecde, M.D., Department of Pharmacolengy and Toxicology, University of Bergen. Armatuer Hansens Hus, N-50121 Hatukeland sykehus, Norwily.

Supported by grants from the Norwegian Rescarch (ouncil, the Nordic Insulin Foundation, and the loutch Prateventicfonds. cysteine and cobalamin, in that order, were the strongest predictors of dictary group. Mean corpuscular volume and It had low discriminative power. We conclude that the determination of methylmalonic acid and total homexysteine represents a sensitive and specific lest for the diagnosis and follow-up of nutritional cobalamin deficiency in infants. Furthermore, the finding of high methylmalonic acid and total homocysteine in plasma of most macrobiotic infants demonstrates a functional cobalamin deficiency in these subjects. (Pediatr Res 36: 194-201, 1994)
Hcy, homocysteinc
MMA, methylmalonic acid
MCV, mean corpuscular volume
(iM, geometric mean
$\mathbf{r}_{s}$, Spearman rank correlation coefficient
OR, odds ratio
CI, confidence interval

Abbreviations

cobalamin deficiency (1). However, recent clinical research has demonstrated the occurrence of cobalamin dysfunction in a large number of patients with normal values for serum cobalamin and no hematologic abnormalities (8-11). Neuropsychiatric disorders may develop even in the absence of anemia and macrocytosis (9). Furthermore, macrocytosis may be masked by concurrent iron deficiency, which is often encountered in vegetarians $(6,12)$.

Because the traditional laboratory tests do not completely discriminate cobalamin-deficient patients from persons with normal cobalamin function, new tests based on measurement of metabolites accumulating during impaired cobalamin function have been developed (13). 
Methionine synthase (EC 2.1.1.13), which remethylates Hcy to methionine, and methylmalonyl-CoA mutase (EC 5.4.99.2), which converts methylmalonyl-CoA to succinyl-CoA, are the only known mammalian enzymes requiring cobalamin as cofactor (14). Inhibition of metabolic flux through these pathways and the resulting increase in the extracellular levels of MMA and Hcy reflect impaired intracellular cobalamin function. This explains the marked elevation of MMA and Hcy in plasma and urine from cobalamin-deficient patients $(10,15-17)$. MMA seems to be a relatively sensitive and specific marker of cobalamin function, whereas Hcy also increases in folate-deficient patients (10). Determination of MMA and Hcy in plasma and serum has been established as a valuable adjunct to the conventional laboratory tests for cobalamin deficiency, especially in cases with subtle or atypical symptoms. Additional attractive features are the relation between elevated metabolite level and therapeutic response and the possibility within 4 to $10 \mathrm{~d}$ to demonstrate the effect from supplementing the deficient vitamin by monitoring a decline in metabolite concentration $(10,17)$.

There are occasional reports on marked elevation of urinary MMA $(2,7,18,19)$ and homocystine $(2,7,18,19)$ in cobalamin-deficient infants of vegetarian mothers, suggesting impairment of intracellular cobalamin function in these patients. Since 1985, techniques for the determination of these metabolites in serum and plasma have been developed and reference levels have been established in adults (20-24). However, there are no data on MMA and Hcy in plasma or serum from healthy or diseased newborns or infants.

In a recent study, the cobalamin and hematologic status was investigated in infants on macrobiotic diets and group-matched omnivorous controls. The macrobiotic infants had very low cobalamin intake (25) and low plasma cobalamin levels combined with several hematologic abnormalities (6). In the present paper, we report on the plasma levels of MMA and Hcy in these macrobiotic infants and in controls. The purpose of this study was 1 ) to obtain the normal levels of these metabolites in healthy infants; 2) to investigate the metabolite levels and thereby the intracellular cobalamin function in macrobiotic infants; and 3) to evaluate the usefulness of MMA and Hcy in plasma for the individual diagnosis of cobalamin deficiency in infancy.

\section{METHODS}

Subjects. The subjects included in this study have been characterized in detail in a previous publication on nutritional status in macrobiotic infants (25). Briefly, blood samples were taken from 50 such infants ( 26 boys) and 57 group-matched controls ( 31 boys) (6). The mothers of the macrobiotic infants had lived on a diet described by Kushi and Jack (26) for at least $3 \mathrm{y}$ and had been feeding their children breast milk from birth onward with later complementary feeding according to macrobiotic princi- ples. Plasma samples for measurement of both MMA and total Hcy were available from 41 macrobiotic infants (22 boys) and 50 controls ( 27 boys). MMA was measured in an additional three samples from macrobiotic infants and four samples from control infants. The mean age of the macrobiotic infants was 16.8 mo (range 11.4-21.9 mo), and that of the controls was 15.8 mo (range 11.1-21.4 mo). Details on food consumption and the study design have been published (25).

Blood sampling and storage. The macrobiotic and control infants were visited at home. Therefore, immediate preparation of the plasma fraction was impossible. Care was taken to visit macrobiotic and control infants in a randomized order. In this way, a systematic difference in time elapsing before centrifugation of whole blood was avoided.

The fasting period was 1 to $4 \mathrm{~h}$ in about $70 \%$ of the children. Blood samples of $4-5 \mathrm{~mL}$ were collected into heparinized glass tubes by venipuncture, carefully mixed, then immediately placed in a cooling box at $4-8^{\circ} \mathrm{C}$, and later stored overnight at $4^{\circ} \mathrm{C}$ in a refrigerator before removal of the blood cells by centrifugation the next day.

The plasma samples were stored at $-20^{\circ} \mathrm{C}$ for about 1.5 mo, then at $-80^{\circ} \mathrm{C}$ for $5 \mathrm{y}$, and finally at $-45^{\circ} \mathrm{C}$ for $6 \mathrm{mo}$. There is evidence that storage of frozen plasma at $-20^{\circ} \mathrm{C}$ for up to $10 \mathrm{y}$ does not change the plasma Hcy level (27).

Analytical methods. All parameters for each infant were assayed in samples collected at the same time point. MMA in plasma was assayed by a method based on HPLC and fluorescence detection; the coefficient of variation of this method is $3-11 \%(23,28)$. Total plasma Hcy and cysteine were determined by a modification (29) of an automated procedure developed for the determination of total Hcy in plasma (30); the coefficient of variation of this method is $<3 \%$ (29). Plasma methionine was determined in deproteinized plasma with an assay based on derivatization with $o$-phthaldialdehyde and fluorescence detection (31). The data for plasma cobalamin, plasma folate, $\mathrm{Hb}$, and $\mathrm{MCV}$ are taken from the study of Dagnelie et al. (6).

Statistical analysis. GM and $\mathrm{GM} \pm 1.96 \mathrm{SD}$ intervals were calculated for MMA, Hcy, cobalamin, methionine, and cysteine in the controls. Because of skew distribution, log-transformation of data was performed before calculation. The data were normally distributed after transformation, as judged by normal probability plots (data not shown). Group GM were compared with the two-sample $t$ test. Correlation between parameters was determined using the $r_{s}$.

In the controls, the 95th percentile for MMA and total Hcy and the 5th percentile for plasma cobalamin were calculated on the basis of the log-transformed data. The 95th percentile for MCV and 5th percentile for $\mathrm{Hb}$ were calculated from the nontransformed data. These values were defined as cutoff values. Metabolite concentrations or MCV above or cobalamin and $\mathrm{Hb}$ levels below the cutoff were regarded as abnormal. 
Logistic regression analysis was used to predict the dietary status (macrobiotic or control). MMA, Hcy, cobalamin, $\mathrm{MCV}$, and $\mathrm{Hb}$ served as candidate predictor variables. The ability of the different tests, used alone or in combination, to predict dietary group was estimated by OR. Two-sided $p$ values less than 0.05 were considered significant.

Analyses were carried out with the BMDP statistical package (BMDP Statistical Software, Inc., Los Angeles, CA).

\section{RESULTS}

Metabolite concentrations in plasma of control infants. MMA, total Hcy, and methionine in plasma from healthy control infants showed skew distribution. The GM for MMA in 50 infants was $0.18 \mu \mathrm{mol} / \mathrm{L}(\mathrm{GM} \pm 1.96 \mathrm{SD}$, $0.06-0.51 \mu \mathrm{mol} / \mathrm{L})$. The GM $(n=50)$ for total Hcy was
$7.6 \mu \mathrm{mol} / \mathrm{L}(5.3-11.0 \mu \mathrm{mol} / \mathrm{L})$ (Table 1). Neither plasma MMA nor total plasma Hcy was significantly related to the age of the infants. Methionine in plasma [6.9 (1.141.9) $\mu \mathrm{mol} / \mathrm{L}$ ] was slightly lower than reported for children with a mean age of 8 y (32). These values and the plasma concentrations for total cysteine and cobalamin in control infants are summarized in Table 1.

Metabolite concentrations in plasma of macrobiotic infants. The concentration of MMA in plasma from macrobiotic infants varied over a wide range $(0.19-15.0 \mu \mathrm{mol} /$ $\mathrm{L})$, and the GM of $1.44 \mu \mathrm{mol} / \mathrm{L}(0.17-12.2 \mu \mathrm{mol} / \mathrm{L}, \mathrm{GM} \pm$ $1.96 \mathrm{SD})$ was 7.5 -fold higher than the mean for controls $(p$ $<0.0001)$. The GM for total Hcy of $13.5(6.8-26.8)$ $\mu \mathrm{mol} / \mathrm{L}$ was increased about 2 -fold compared with controls $(p=0.0014$; Table 1, Fig. 1). The plasma level of methionine was not significantly different between mac-

Table 1. Plasma levels of metabolites and cobalamin in macrobiotic and healthy control infants*

\begin{tabular}{lcccccc}
\hline & MMA $(\mu \mathrm{mol} / \mathrm{L})$ & Hcy $(\mu \mathrm{mol} / \mathrm{L})$ & Cobalamin $(\mathrm{pmol} / \mathrm{L})$ & Cysteine $(\mu \mathrm{mol} / \mathrm{L})$ & Methionine $(\mu \mathrm{mol} / \mathrm{L})$ \\
\hline Macrobiotics $(n=41)$ & $1.44(0.17-12.15)$ & $13.5(6.8-26.8)$ & $141.4(58.8-339.7)$ & $163.6(118.7-225.6)$ & $6.0(1.5-23.8)$ \\
Controls $(n=50)$ & $0.18(0.06-0.51)$ & $7.59(5.3-11.0)$ & $398.7(193.6-820.7)$ & $183.8(144.7-233.4)$ & $6.9 \dagger(1.1-41.9)$ \\
$p$ value & $<0.0001$ & 0.0014 & $<0.0001$ & 0.0001 & 0.44 &
\end{tabular}

* Data are given as $\mathrm{GM}$ and, in parentheses, $\mathrm{GM} \pm 1.96 \mathrm{SD}$.

$\dagger n=49$.

$\ddagger$ Two-sample $t$ test.
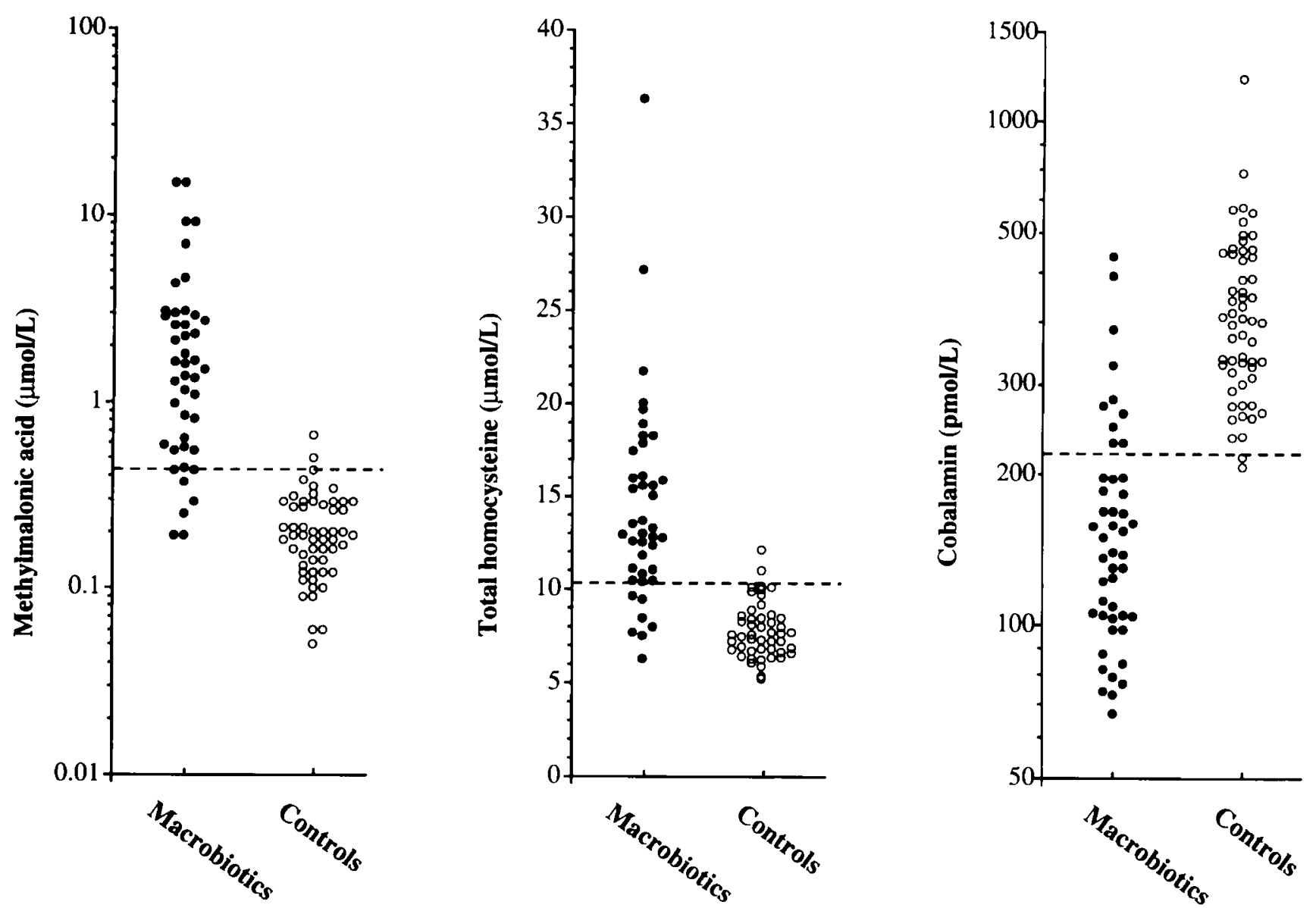

Figure 1. Concentrations of MMA, total Hcy, and cobalamin in plasma from macrobiotic infants $(n=41)$ and control infants $(n=50)$. The horizontal dashed lines represent the upper 95th percentile for methylmalonic and total Hcy and the lower 5th percentile for cobalamin in plasma from control infants. 
robiotic infants and controls. Total cysteine was slightly but significantly $(p=0.0001)$ reduced in macrobiotic infants (Table 1).

Relation between plasma metabolite concentrations and other parameters. MMA in plasma was inversely correlated $\left(r_{\mathrm{s}}=-0.765, p<0.001\right)$ with plasma cobalamin. A plot of cobalamin versus MMA gave a hyperbolic relation, and marked elevation of MMA was observed at levels of plasma cobalamin below about $220 \mathrm{pmol} / \mathrm{L}$ (Fig. 2). Also, total Hcy showed an inverse correlation $\left(r_{\mathrm{s}}=-0.741 ; p<0.001\right)$ with plasma cobalamin (Fig. 2). The inverse correlation between MCV and plasma co- balamin, published previously $(6)$, was weaker $\left(r_{\mathrm{s}}=\right.$ $-0.313 ; p<0.01)$. There was a strong positive correlation $\left(\mathrm{r}_{\mathrm{s}}=0.772 ; p<0.001\right)$ between MMA and total Hcy in plasma (Fig. 3), but in a few cases, only MMA (six cases) or total Hcy (seven cases) was elevated (Fig. 3). Plasma folate was higher in the macrobiotic group than in the control group (6) and there was a tendency that high plasma folate levels were associated with high concentrations of total plasma Hcy in macrobiotic infants $(r=$ $0.252 ; p=0.1)$, but not in controls $(r=-0.207 ; p=0.15)$ (Fig. 4). However, these relations were not statistically significant.

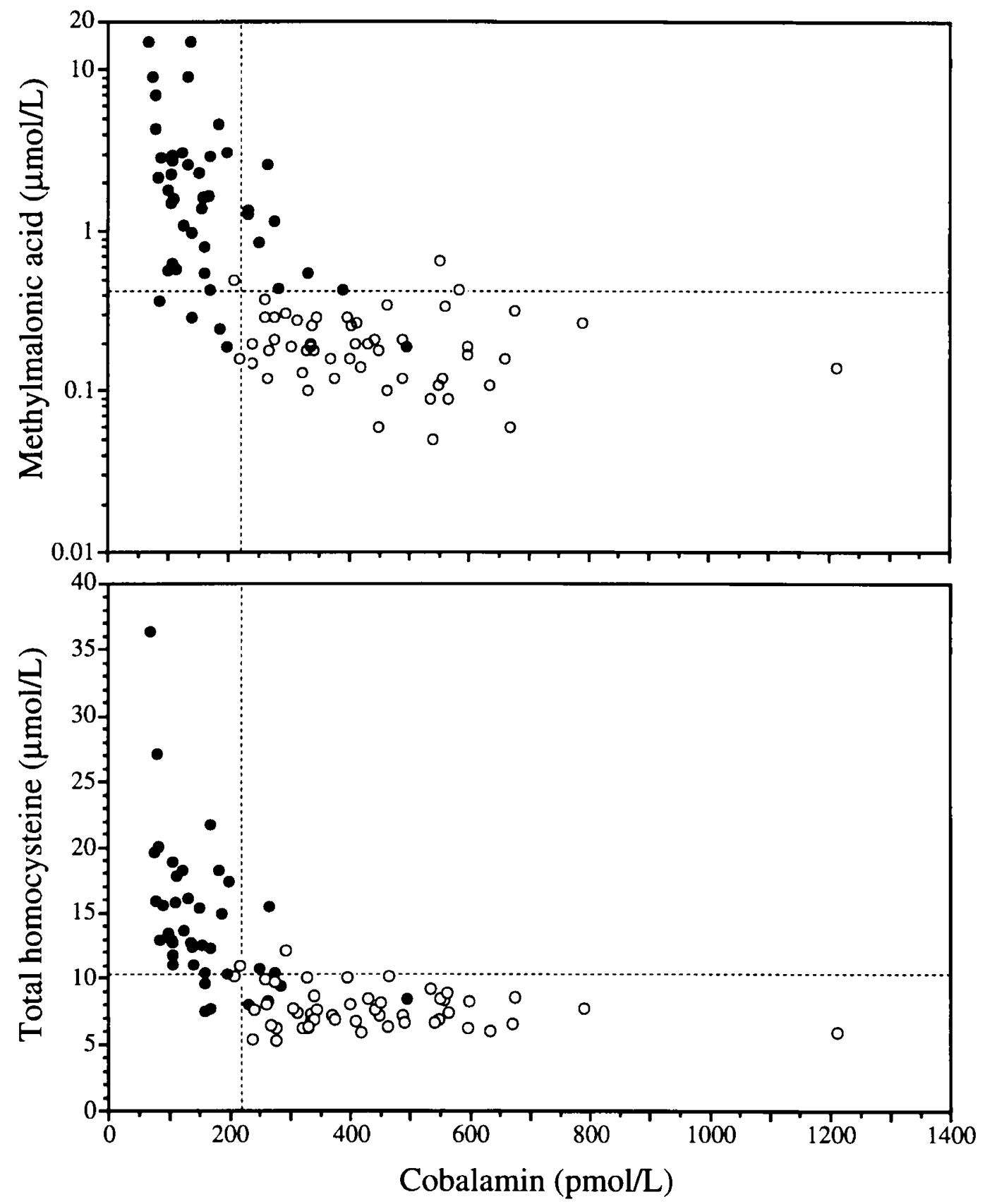

Figure 2. Correlation between cobalamin, MMA, and total Hcy in plasma. The filled symbols show the data for the macrobiotic infants, and the open symbols show the data for the control infants. There is a significant $(p<0.001)$ negative correlation between cobalamin and MMA $\left(r_{s}=\right.$ $-0.765)$ and between cobalamin and total $\mathrm{Hcy}\left(r_{s}=-0.741\right)$. The dashed lines represent the upper 95th percentile for MMA and Hcy and the lower 5 th percentile for cobalamin in plasma from control infants. 


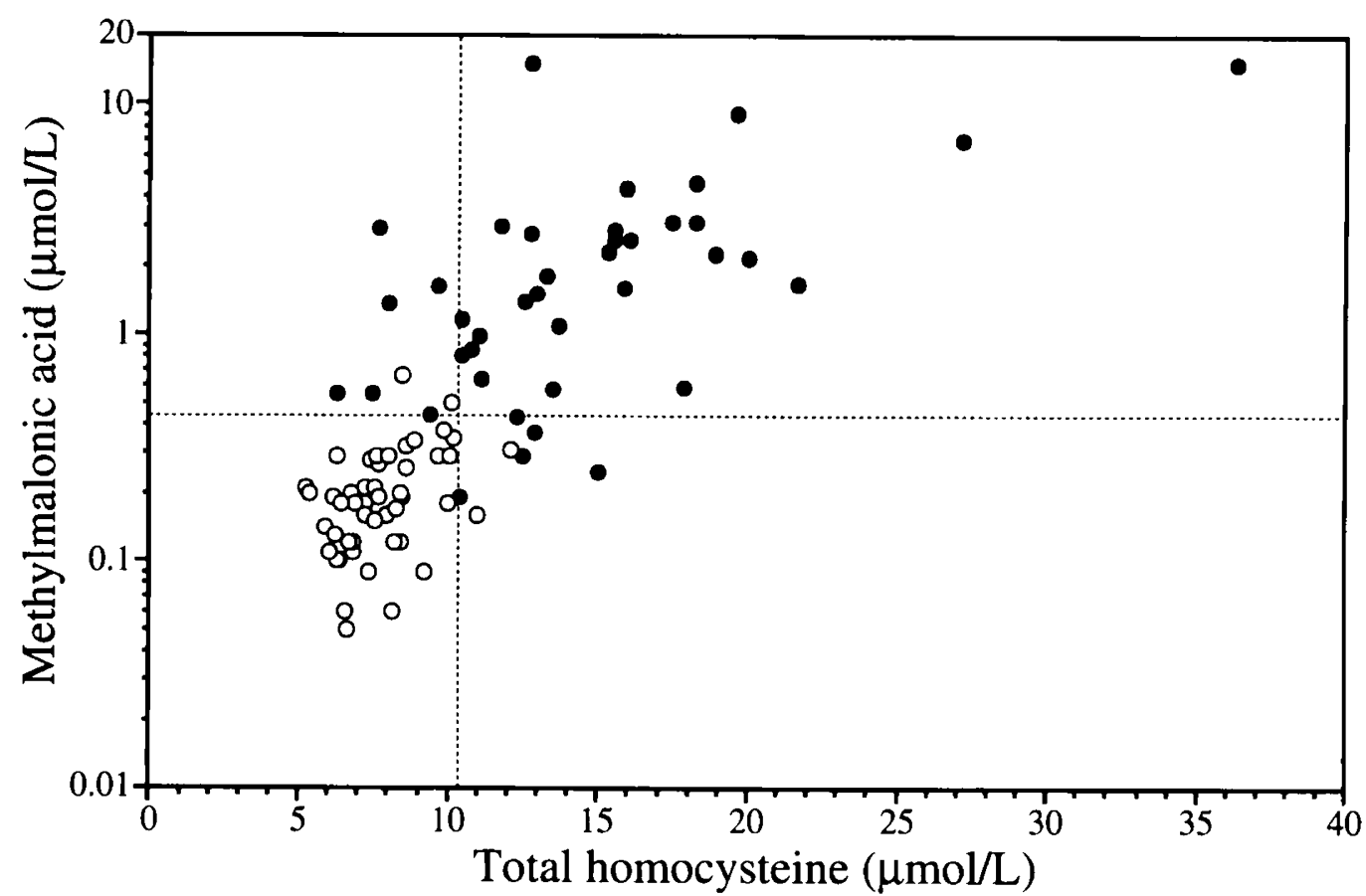

Figure 3. Correlation between total Hcy and MMA in plasma. The filled symbols show the data for the macrobiotic infants, and the open symbols show the data for the control infants. There is a significant $(p<0.001)$ positive correlation between these two parameters $\left(r_{\mathrm{s}}=0.772\right)$. The dashed lines represent the upper 95 th percentiles for total Hcy and MMA in plasma from control infants.

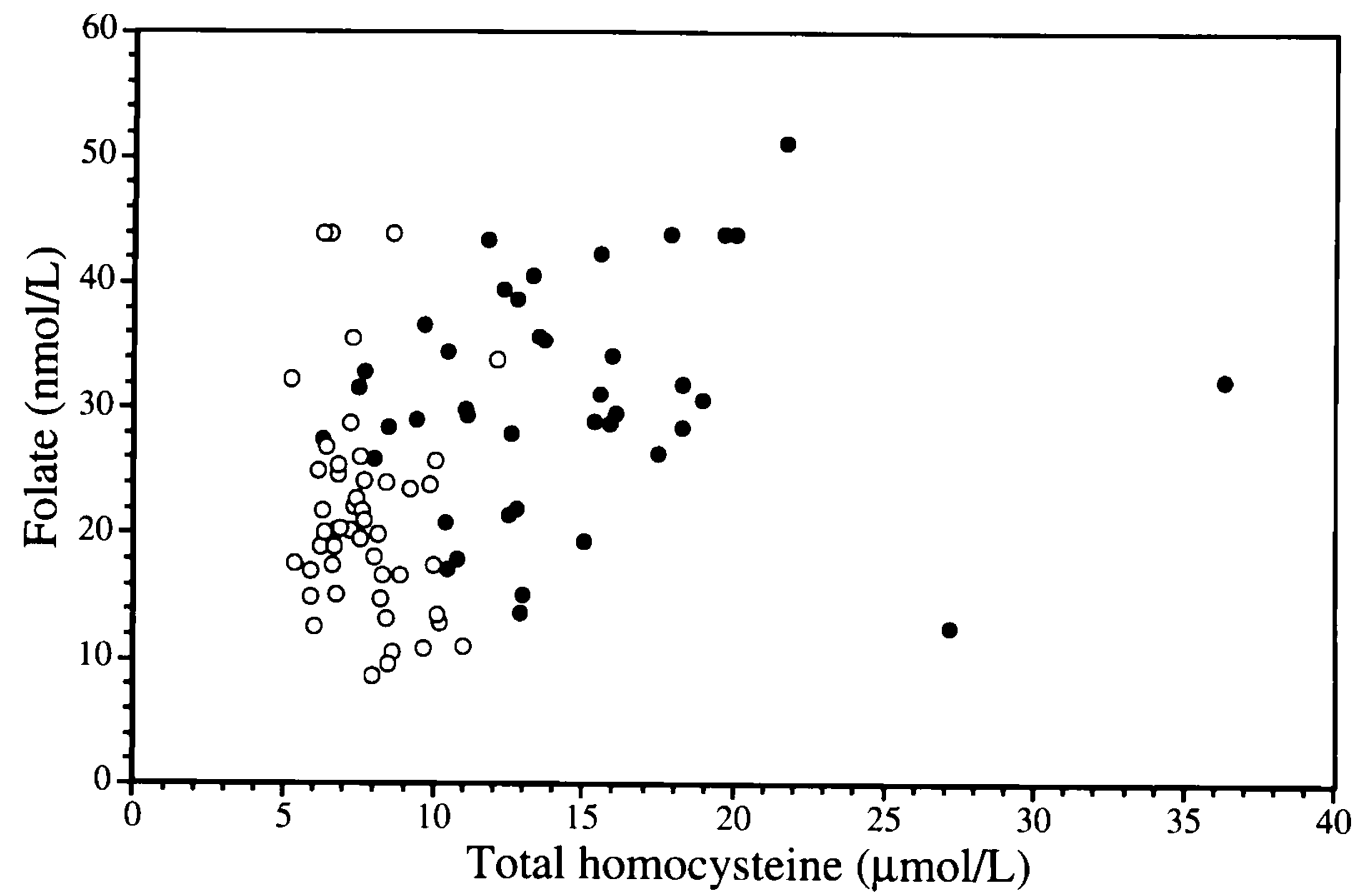

Figure 4. Correlation between total plasma Hcy and serum folate in macrobiotic infants and in control infants. The data points for the macrobiotic infants are given as filled symbols. There was a trend $(p=0.1)$ toward a positive correlation between total Hcy and folate $\left(\mathrm{r}_{\mathrm{s}}=0.252 ; p=0.1\right)$. The open symbols are the data points for the controls. A trend $(p=0.15)$ toward a negative correlation between these two parameters was detected in this population $\left(\mathrm{r}_{\mathrm{s}}=-0.207 ; p=0.15\right)$.

Discrimination between macrobiotic infants and controls. In 91 infants, values were obtained for MMA, Hcy, and cobalamin; 41 infants belonged to the macrobiotic group and 50 infants were controls. In 26 subjects, all macrobiotic infants, all three parameters were abnormal. In another 13 macrobiotic and two control infants, two parameters were abnormal. The sensitivity of
MMA as a test to predict the macrobiotic group was 35 of $41(85.4 \%)$. The corresponding values for Hcy and cobalamin were 34 of $41(83 \%)$ and 34 of $41(83 \%)$, respectively (Fig. 1, Table 2). The simultaneous use of MMA and Hcy (one or more positive), increases the sensitivity to $98 \%$ without loss of specificity (92\%) (Fig. 3). 
Table 2. Fractions of abnormal plasma levels of metabolites, cobalamin, $M C V$, and $H b$ in macrobiotic infants

\begin{tabular}{lcc}
\hline & \multicolumn{2}{c}{ Macrobiotic infants } \\
\cline { 2 - 3 } Parameter & Fraction abnormal* & $\%$ \\
\hline MMA & $35 / 41$ & 85.4 \\
Total Hcy & $34 / 41$ & 83.0 \\
Cobalamin & $34 / 41$ & 83.0 \\
MCV & $9 / 41$ & 22.0 \\
Hb & $5 / 41$ & 12.2 \\
\hline
\end{tabular}

* Abnormal levels are defined as values above the 95 th percentile of healthy control infants and are $>0.43 \mu \mathrm{mol} / \mathrm{L}$ for $\mathrm{MMA},>10.35 \mu \mathrm{mol} / \mathrm{L}$ for total Hcy, and $>88.23 \mathrm{fL}$ for MCV. For cobalamin and Hb, abnormal levels are defined as below the 5 th percentile of healthy control infants and are $<218 \mathrm{pmol} / \mathrm{L}$ and $<112.7 \mathrm{~g} / \mathrm{L}$, respectively.

Logistic regression was used to predict the dietary group from the values of MMA, total Hcy, cobalamin, $\mathrm{MCV}$, and $\mathrm{Hb}$. The single strongest predictor of dietary group was MMA with an OR of $140(95 \% \mathrm{CI}=26.1-752)$. This means that the odds of belonging to the macrobiotic diet group were 140 times higher with an abnormal compared with a normal plasma MMA level. Total Hcy and cobalamin were also strong predictors, whereas $\mathrm{MCV}$ and $\mathrm{Hb}$ discriminated poorly between the diet groups (Table 3).

When all five parameters were included in the regression analysis simultaneously (multivariate analysis), again plasma MMA $(\mathrm{OR}=109 ; 95 \% \mathrm{CI}=7.0-1720)$ was superior to total Hcy $(\mathrm{OR}=51.3 ; 95 \% \mathrm{CI}=2.1-1290)$ and plasma cobalamin $(\mathrm{OR}=3.5 ; 95 \% \mathrm{CI}=0.25-50.8)$ as a predictor.

We also compared the ability of plasma MMA, total $\mathrm{Hcy}, \mathrm{MCV}$, and $\mathrm{Hb}$ to increase the discriminative power afforded by plasma cobalamin (bivariate analysis). MMA had a higher OR $(72.2 ; 95 \% \mathrm{CI}=9.07-575)$ than plasma Hcy $(22.2 ; 95 \% \mathrm{CI}=3.34-147)$. The corresponding OR for $\mathrm{MCV}$ and $\mathrm{Hb}$ were $3.21(95 \% \mathrm{CI}=0.38-27.1)$ and

Table 3. Logistic regression analysis of plasma levels of metabolites, cobalamin, $\mathrm{MCV}$, and $\mathrm{Hb}$ as predictors of dietary group*

\begin{tabular}{lccl}
\hline & \multicolumn{3}{c}{ OR } \\
\cline { 2 - 4 } Parameter & Univariate $\dagger$ & Multivariate $\ddagger$ & \multicolumn{1}{c}{ Bivariate $\$$} \\
\hline \multirow{2}{*}{ MMA } & $140.0(26.1-752)$ & $109(7.0-1720)$ & $72.2(9.1-575)$ \\
& $p<0.0001$ & $p=0.001$ & $p=0.001$ \\
Total Hcy & $117.0(22.3-610)$ & $51.3(2.1-1290)$ & $22.2(3.3-147)$ \\
& $p<0.0001$ & $p=0.017$ & $p=0.0169$ \\
Cobalamin & $117.0(22.3-610)$ & $3.5(0.25-50.8)$ & \\
& $p<0.0001$ & $p=0.35(\mathrm{NS}) \|$ & \\
MCV & $4.41(1.09-17.9)$ & $4.85(0.13-180)$ & $3.21(0.38-27.1)$ \\
& $p=0.04$ & $p=0.39(\mathrm{NS}) \|$ & $p=0.28(\mathrm{NS}) \|$ \\
Hb & $1.60(0.39-6.50)$ & $2.5(0.04-174.8)$ & $0.69(0.05-8.9)$ \\
& $p=0.51(\mathrm{NS}) \|$ & $p=0.67(\mathrm{NS}) \|$ & $p=0.78(\mathrm{NS}) \|$ \\
\hline
\end{tabular}

* The values are given as OR with $95 \% \mathrm{CI}$ in parentheses. The same cutoff levels as in Table 2 were included in the analysis.

† Only one parameter is included in the analysis.

$\ddagger$ All parameters are included simultaneously.

$\$$ Cobalamin and one additional parameter are included.

$\| p$ values $>0.05$ were regarded as not significant.
$0.69(95 \% \mathrm{CI}=0.05-8.9)$, respectively, which were not statistically significant (Table 3 ).

\section{DISCUSSION}

Blood sampling and reference values. For organizational reasons, the whole-blood samples were stored overnight in a refrigerator $\left(4^{\circ} \mathrm{C}\right)$ before centrifugation the next day. The time before blood cells were removed was maximally $24 \mathrm{~h}$. It is unlikely that this affected the concentration of MMA in plasma, because even storage of whole blood for $24 \mathrm{~h}$ at room temperature has been shown to be without effect (33). This is corroborated by the observation that the reference interval obtained for the healthy control infants in this study $[0.06-0.51(\mathrm{GM} \pm 1.96 \mathrm{SD}) \mu \mathrm{mol} / \mathrm{L}]$ equals that established for adults (20-23).

In whole blood, there is a time- and temperaturedependent production of Hcy. The amounts are independent of the plasma Hcy concentration (29). The relative increase upon storage is therefore larger at low than at high Hcy concentrations. Based on data on Hcy production in whole blood from adults (29), one might expect an artificial increase of about $1 \mu \mathrm{mol} / \mathrm{L}$ during storage for 24 $h$ at $4-8^{\circ} \mathrm{C}$. However, the increase in Hcy is limited, because the values for total Hcy in infants aged 10-24 mo obtained in the present study (GM $7.59 \mu \mathrm{mol} / \mathrm{L})$ are of the same magnitude as the plasma concentrations previously reported in 10 children aged $6.2 \pm 3.5$ y (34) and are lower than the normal level published for adults (35).

In studies based on stored samples, blood sampling and processing often do not fully comply with the strict procedures recommended for the determination of Hcy (24). In general, a moderate increase in plasma Hcy occurring before separation of blood cells does not undermine the diagnostic value of plasma Hcy as an indicator of vitamin deficiency, inasmuch as these conditions induce a marked elevation of the plasma Hcy level (24). In casecontrol studies, such error is partly adjusted for by identical sample handling for the investigated population and the reference group. This was accomplished in the present study. Furthermore, the variability in plasma Hcy among the 50 control infants $(95 \% \mathrm{CI}=5.3-11.0 \mu \mathrm{mol} / \mathrm{L}$; Table 1) is less than the variability of plasma Hcy in 3000 healthy men sampled under optimal conditions (24). This strongly suggests a uniform procedure for sample handling in the present study. The limited increase in plasma Hcy that probably takes place before the removal of the blood cells would reduce the relative difference in plasma Hcy between macrobiotic infants and controls. This results in an underestimation of the diagnostic sensitivity and specificity of plasma Hcy.

Cobalamin function and metabolite response in macrobiotic infants. The hematologic parameters showed subtle differences between macrobiotic infants and controls, and there was considerable overlap between the groups. $\mathrm{Hb}$ was not significantly different between the groups. Hematocrit and red blood cell count were lower, and $\mathrm{MCV}$, mean corpuscular $\mathrm{Hb}$, and mean corpuscular $\mathrm{Hb}$ 
concentration were higher in macrobiotic infants compared with controls. No significant neurologic dysfunction could be demonstrated upon clinical examination, but the macrobiotic children showed retardation of growth and psychomotor development compared with the omnivorous controls (36). Plasma cobalamin was markedly reduced in macrobiotic infants, suggesting impaired cobalamin function in most macrobiotic infants.

In adults, MMA in serum and plasma is a more specific marker for cobalamin function than total Hcy, which is also elevated in folate-deficient patients and in some other diseases (24). MMA also seems to have a somewhat higher sensitivity than total Hcy (10). Of particular importance is the finding of elevated serum MMA or total Hcy in $94 \%$ of patients with an objective biochemical response to cobalamin supplementation (11). In another study on clinically confirmed cobalamin-deficient patients, a test sensitivity of $97 \%$ and a specificity of $91 \%$ were reported for serum MMA (37).

Plasma and serum levels of MMA and total Hcy probably reflect intracellular cobalamin function in infants in the same way as in adults. This is supported by the observed relation between plasma cobalamin and metabolite concentrations, as depicted in Figure 2. Elevation of MMA, total Hcy, or both in plasma (Fig. 3) is observed in 40 of 41 macrobiotic infants (Table 2). This finding shows that low plasma cobalamin is associated with impaired cobalamin function in a much larger portion of these infants than might be expected from the occurrence of hematologic abnormalities (6).

It has been shown previously that plasma folate levels in the macrobiotic infants were markedly elevated and negatively related to plasma cobalamin. Regression analysis suggested that the deleterious effect of low plasma cobalamin on hematologic parameters was stronger at high folate concentrations. Thus, the high folate in macrobiotic infants is probably not caused by high folate intake but is a consequence of cobalamin deficiency (6). Our data, which show a positive (although not statistically significant) relation between plasma folate and total Hcy in macrobiotic infants (Fig. 4), are in contrast to the negative correlation between these two parameters found in a population of adults with variable folate status (24). This indicates impaired tissue utilization of folate during cobalamin deficiency. Elevation of both folate and total Hcy in plasma may reflect an impaired function of the cobalamin-dependent methionine synthase resulting in accumulation of the substrates, Hcy, and 5-methyltetrahydrofolate.

Evaluation of MMA and total Hcy in plasma as diagnostic tests. To evaluate plasma MMA and total plasma Hcy as tests to reveal cobalamin deficiency in individual infants, an objective measure of functional cobalamin deficiency is required. Clinical improvement and/or normalization of metabolite concentrations after cobalamin supplementation may be regarded as independent measures of cobalamin deficiency $(11,37)$. However, the clinical and hematologic symptoms are often subtle, and an interven- tion was performed in only a limited number of infants included in the present study $(38,39)$. Therefore, our data do not allow an independent discrimination between healthy and cobalamin-deficient infants.

Because no clinical sign or independent biochemical test discriminates between cobalamin-deficient and healthy infants, we chose dietary group as an independent indicator of cobalamin deficiency in these infants, assuming that cobalamin-deficient and nondeficient infants coincide with the macrobiotic group and control group, respectively. To our best knowledge there do not exists reports on nutritional cobalamin deficiency in infants born to healthy omnivorous mothers, suggesting that this is extremely rare. In contrast, based on analytical data of plant foods included in the macrobiotic diet, the estimated cobalamin intake in these infants is extremely low (25). Furthermore, elevated MCV was previously reported in the macrobiotic group (6). Importantly, the rise in MCV was not limited to just a few macrobiotic infants, but the macrobiotic group as a whole showed an elevation of MCV resulting in a shift of the cumulative frequency curve of MCV toward higher values compared with the control group. This supports the view that (even though MCV values were apparently within "normal" range for part of the macrobiotic group) the macrobiotic group as a whole is a cobalamin-deficient population. These considerations justify the assumption that group membership (i.e. macrobiotic versus omnivorous group) is a good indicator of cobalamin deficiency versus no deficiency.

MMA, total Hcy and cobalamin measurements identified the macrobiotic infants with high sensitivity $(>82 \%)$ (Table 2), but regression analyses showed that plasma MMA had the highest discriminative power followed closely by total plasma Hcy and plasma cobalamin, both when analyzed separately (univariate analysis) and simultaneously (multivariate analysis). MCV and, even more so, $\mathrm{Hb}$ were insensitive and unspecific in diagnosing cobalamin deficiency in the individual infant (Table 3), even though MCV was useful as an indicator of cobalamin deficiency in a population (6). We also evaluated which test added the most information when cobalamin value was already included in the predictive equation (bivariate analysis), and MMA was also superior in this respect (Table 3 ).

The OR for elevated MMA and total Hcy were different, and MMA, Hcy, and cobalamin provide independent predictive information (Table 3 ). This is related to the observation that in some macrobiotic infants, the plasma level of only one metabolite is elevated, as demonstrated in Figure 3. Thus, determination of the plasma levels of both MMA and total Hcy may discriminate better between the groups than one parameter alone, and the simultaneous use of MMA and Hcy increases the test sensitivity without loss of specificity (sensitivity $98 \%$; specificity $92 \%$ ).

Plasma MMA, in contrast to total Hcy, is a specific marker of cobalamin function (10), but adding total Hcy 
to the test battery may differentiate nutritional cobalamin deficiency from "benign" methylmalonic aciduria (40) or some rare congenital cobalamin mutations affecting methylmalonyl CoA mutase (1).

Conclusion. The marked elevation of both plasma MMA and total Hcy in macrobiotic infants compared with controls presents further evidence in favor of a functional cobalamin deficiency in a vast majority of these infants. Determination of metabolite concentrations in plasma and serum discriminates most cobalamindeficient infants from healthy controls and represents a useful adjunct to the conventional diagnostic tests. The analysis can be carried out in samples already collected for the cobalamin assay, and no urine collection or determination of creatinine is required. Finally, normalization of metabolite levels in plasma is an objective and early measure of therapeutic response. These are features of importance for a rapid diagnosis as well as for a close follow-up of nutritional cobalamin deficiency in infants.

Acknowledgments. The technical assistance provided by Gry Kvalheim and Elfrid Blomdal is highly appreciated.

\section{REFERENCES}

1. Davis RE 1985 Clinical chemistry of vitamin B12. Adv Clin Chem 24:163-216

2. Miller DR. Specker BL., Ho MI. Norman EJ 1991 Vitamin-B-12 status in macrobiotic community. Am J Clin Nutr 53:524-529

3. Higginbottom MC, Sweetman L, Nyhan WL 1978 A syndrome of methylmalonic aciduria, homocystinuria, megaloblastic anemia and neurological abnormalities in a vitamin B12-deficient breastfed infant of a strict vegetarian. N Engl J Med 299:317-323

4. Sklar R 1986 Nutritional vitamin B12 deficiency in a breast-fed infant of vegan-diet mother. Clin Pediatr 25:219-221

5. Specker BL, Miller D, Norman EJ, Greene H, Hayes KC 1988 Increased urinary methylmalonic acid excretion in breast-fed infants of vegetarian mothers and an identification of an acceptable source of vitamin B12. Am J Clin Nutr 47:89-92

6. Dagnelie PC, van Staveren WA, Vergote FJVRA, Dingjan PG, van den Berg $\mathrm{H}$, Hautvast JGAJ 1989 Increased risk of vitamin B-12 and iron deticiency in infants on macrobiotic diets. Am J Clin Nutr 50:818-824

7. Kuhne T, Bubl R, Baumgartner R 1991 Maternal vegan diet causing a serious infantile neurological disorder due to vitamin-B12 deficiency. Eur J Pediatr $150: 205-208$

8. Carmel R, Sinow RM, Karnaze DS 1987 Atypical cobalamin deficiency: subtle biochemical evidence of deficiency is commonly demonstrated in patients without megaloblastic anemia and is often associated with proteinbound cobalamin malabsorption. J Lab Clin Med 109:454-463

9. Lindenbaum J, Healton EB, Savage DG, Brust JCM, Garrett TJ, Podell ER Marcell PD. Stabler SP, Allen RH 1988 Neuropsychiatric disorders caused by cobalamin deficiency in the absence of anemia or macrocytosis. $N$ Engl J Med 318:1720-1728

10. Lindenbaum J, Savage DG, Stabler SP, Allen RH 199) Diagnosis of cobalamin deficiency. II. Relative sensitivities of serum cobalamin, methylmalonic acid, and total homocysteine concentrations. Am J Hematol 34:99-107

11. Stabler SP, Allen RH, Savage DG, Lindenbaum J 199) Clinical spectrum and diagnosis of cobalamin deficiency. Blood $76: 871-881$

12. Carmel $R 1990$ Subtle and atypical cobalamin deficiency states. Am J Hematol 34:108-114

13. Lindenbaum J, Stabler SP, Allen RH 1988 New assays for cobalamin deficiency getting better specificity. Lab Manag 26:41-44

14. Beck WS 1990) Cobalamin as coenzyme: a twisting trail of research. Am Hematol 34:83-89

15. Brattström L. Israelsson B. Lindgärde F, Hultherg B 1988 Higher total plasma homocysteine in vitamin B12 deficiency than in heterozygosity for homocystinuria due to cystathionine $\beta$-synthase deficiency. Metabolism $37: 175-178$
16. Rasmussen K, Moelby L. Jensen MK 1989 Studies on methylmalonic acid in humans. II. Relationship between concentrations in serum and urinary excretion. and the correlation between serum cobalamin and accumulation of methylmalonic acid. Clin Chem 35:2277-2280)

17. Allen RH, Stabler SP, Savage DG, Lindenbaum J 199) Diagnosis of cobalamin deficiency. 1. Usefulness of serum methylmalonic acid and total homocysteine concentrations. Am J Hematol 34:9(2-98

18. Specker BL. Black A, Allen L, Morrow F 1990 Vitamin B-12: low milk concentrations are related to low serum concentrations in vegetarian women and to methylmalonic aciduria in their infants. Am J Clin Nutr 52:1073-1076

19. Michaud JL, Lemieux B, Ogier H, Lambert MA 1992 Nutritional vitamin-B12 deficiency: two cases detected by routine newborn urinary screening. Eur J Pediatr 151:218-220

20. Stabler SP, Marcell PD, Podell ER, Allen RH. L indenbaum J 1986 Assay of methylmalonic acid in the serum of patients with cobalamin deficiency using capillary gas chromatography-mass spectrometry. J Clin Invest 77:1606-1612

21. Rasmussen K 1989 Solid-phase sample extraction for rapid determination of methylmalonic acid in serum and urine by a stable-isotope dilution method. Clin Chem 35:260)-264

22. Rasmussen K, Moller J, Ostergaard K, Kristensen OM, Jensen J 1990 Methylmalonic acid concentrations in serum of normal subjects: biological variability and effect of oral 1 -isoleucine loads before and after intramuscular administration of cobalamin. Clin Chem 36:1295-1299

23. Schneede J, Ueland PM 1993 Automated assay for methylmalonic acid in serum and urine by derivatization with 1-pyrenyldiazomethane, liquid chromatography and fuorescence detection. Clin Chem 39:392-394

24. Ueland PM, Refsum H, Stabler SP, Malinow MR, Andersson A, Alien RH 1993 Total homocysteine in plasma or serum. Methods and clinical applica. tions. Clin Chem 39:1764-1779

25. Dagnelie PC, Van Staveren WA, Verschuren SAJM, Hautvast JGAJ 1989 Nutritional status of infants on macrobiotic diets aged 4 to 18 months and matched omnivorous control infants: a population-based mixed-longitudinal study. I. Weaning pattern, energy and nutrient intake. Eur J Clin Nutr $43: 311-323$

26. Kushi M, Jack A 1987 The Book of Macrobiotics. The Universal Way of Health, Happiness, and Peace. Japan Publications, Boston

27. Israelsson B, Brattstrom L, Refsum H 1993 Homocysteine in frozen plasma samples: a short cut to establish hyperhomocysteinaemia as a risk factor for arteriosclerosis. Scand J Clin Lab Invest 53:465-469

28. Schneede J. Ueland PM 1992 The formation in an aqueous matrix, properties and chromatographic behavior of 1-pyrenyldiazomethane derivatives of methylmalonic acid and other short chain dicarboxylic acids. Anal Chem 64:315-319

29. Fiskerstrand T, Refsum H, Kvalheim G, Ueland PM 1993 Homocysteine and other thiols in plasma and urine: automated determination and sample stability. Clin Chem 39:26.3-271

30. Refsum H, Ueland PM, Svardal AM 1989 Fully automated fluorescence assay for determining total homocysteine in plasma. Clin Chem 35:1921-1927

31. Krishnamurti CR, Heindze AM, Galzy G 1984 Application of reversed-phase high-performance liquid chromatography using pre-column derivatization with o-phthaldialdehyde for the quantitative analysis of amino acids in adult and fetal sheep plasma, animal feeds and tissues. J Chromatogr 315:321-331

32. Gregory DM, Sovetts D. Clow CL, Scriver CR 1986 Plasma free amino acid values in normal children and adolescents. Metabolism 35:967-969

33. Marcell PD, Stabler SP. Podell ER, Allen RH 1985 Quantitation of methylmalonic acid and other dicarboxylic acids in normal serum and urine using capillary gas chromatography-mass spectrometry. Anal Biochem 150:58-66

34. Refsum H, Wesenberg F, Ueland PM 1991 Plasma homocysteine in children with acute lymphoblastic leukemia. Changes during a chemotherapeutic regimen including methotrexate. Cancer Res 51:828-835

35. Andersson A. Brattström L, Israelsson B, Isaksson A, Hamfelt A, Hultberg B 1992 Plasma homocysteine before and after methionine loading with regard to age, gender, and menopausal status. Eur J Clin Invest 22:79-87

36. Dagnelie PC, Van Staveren WA, Vergote FJVRA, Burema J, Van't Hof MA Van Klaveren JD, Hautvast JGAJ 1989 Nutritional status of infants on macrobiotic diets aged 4 to 18 months and matched omnivorous contro infants: a population-based mixed-longitudinal study. II. Growih and psychomotor development. Eur J Clin Nutr 43:325-338

37. Moelby L, Rasmussen K, Jensen MK, Pedersen KO 199) The relationship between clinically confirmed cobalamin deficiency and serum methylmalonic acid. J Intern Med 228:373-378

38. Dagnelie PC, Van Staveren WA 1991 Vitamin B12 in seaweed appears not to he bio-available. Am J Clin Nutr 53:695-697

39. Dagnelie PC, Van Staveren WA, Vergote FJVRA 199) Nutritional interven tion and follow-up study of children aged 1-2 years on macrobiotic diets. Ned Tijdschr Geneeskd 134:341-345

4). Tuchman M, Mccann MT, Thompson MM, Tsai MY, Giguere R, Lemieux B 1992 Screening urine of 3-week-old newborns: transient methylmalonic and hydroxyphenyllactic aciduria. Biochem Med Metab Biol 48:64-68 